\title{
Build-up Approach to Updating the Mock Quiet Spike ${ }^{\mathrm{TM}}$ Beam Model
}

\author{
Claudia Y. Herrera ${ }^{1}$ and Chan-gi Pak ${ }^{2}$ \\ NASA Dryden Flight Research Center Edwards, CA
}

\begin{abstract}
A crucial part of aircraft design is ensuring that the required margin for flutter is satisfied. A trustworthy flutter analysis, which begins by possessing an accurate dynamics model, is necessary for this task. Traditionally, a model was updated manually by fine tuning specific stiffness parameters until the analytical results matched test data. This is a time consuming iterative process. NASA Dryden Flight Research Center has developed a Mode Matching Code to execute this process in a more efficient manner. Recently, this code was implemented in the F-15B/Quiet Spike ${ }^{\mathrm{TM}}$ model update. A build-up approach requiring several ground vibration test configurations and a series of model updates was implemented in order to determine the connection stiffness between aircraft and test article. The Mode Matching Code successfully updated various models for the F-15B/Quiet Spike ${ }^{\mathrm{TM}}$ project to within $1 \%$ error in frequency and the modal assurance criteria values ranged from $88.51 \%$ $99.42 \%$.
\end{abstract}

$\begin{array}{lll} & & \text { Nomenclature } \\ C A D & =\text { computer aided design } & \text { Center of gravity } \\ C G & =\text { concentrated mass element } \\ C O N M 2 & =\text { Dryden Flight Research Center } \\ D F R C & =\text { Finite Element } \\ F E & =\text { ground vibration test } \\ G V T & =\text { moment of inertia } \\ I_{k k} & =\text { objective function } \\ J & =\text { Modal Assurance Criteria } \\ M A C & =\text { National Aeronautics and Space Administration } \\ N A S A & =\text { Quiet Spike }\end{array}$

\section{Introduction}

When a new aircraft is designed or a modification is done to an existing aircraft, the aeroelastic properties of the aircraft need to be examined to ensure the aircraft is flight worthy. Evaluating the aeroelastic properties of a new or modified aircraft can include performing a variety of analyses, such as modal and flutter analyses. In order to produce accurate results from these analyses, it is imperative to work with FE models that have been validated by or correlated to GVT data. Updating an analytical model using measured data is a challenge in the area of structural dynamics. The analytical model update process encompasses a series of optimizations that match analytical frequencies and mode shapes to the measured modal characteristics of a structure. In the past, the method used to update a model to test data has been 'trial and error.' This is an inefficient method - running a modal analysis, comparing the analytical results to the GVT data, manually modifying one or more structural parameters (mass, CG, inertia, area, etc.), rerunning the analysis, and comparing the new analytical modal characteristics to the GVT modal data. If the match is close enough (close enough defined by the analyst's updating requirements), then the updating process is finished. If the match does not meet updating requirements, then the parameters are changed again and the process is repeated. Clearly, this manual optimization process is highly inefficient for large FE models and/or a large number of structural parameters.

\footnotetext{
${ }^{1}$ Aerospace Engineer, Structural Dynamics Group, Aerostructures Branch, AIAA Member

${ }^{2}$ Group Lead, Structural Dynamics Group, Aerostructures Branch, AIAA Member
} 
NASA DFRC has developed, in-house, a Mode Matching Code that automates the above-mentioned optimization process. Moreover, it facilitates the development of reduced order models, which simplify analyses and therefore, reduce computation time. DFRC's in-house Mode Matching Code reads mode shapes and frequencies that were measured from a GVT to create the target model. It also reads the current analytical model as the 'initial guess,' as well as the variable parameters and their upper and lower limits as assigned by the analyst. It performs a modal analysis on this initial model and modifies its variable properties to create an updated model that has similar mode shapes and frequencies to those of the target model. The Mode Matching Code outputs frequencies and MAC values that allow for the quantified comparison of the updated model versus the target model.

The Mode Matching Code assists in determining the airworthiness of the aircraft flown at DFRC. Recently, this code was implemented on a project flown on the DFRC F-15B supersonic flight testing platform, the Gulfstream Quiet Spike ${ }^{\mathrm{TM}}(\mathrm{QS})$. This code enabled the build-up approach that was necessary due to test article availability to be implemented and allowed for progress to be made in the analyses even without the flight hardware.

The objective of this work was to use the Mode Matching Code to determine the connection stiffness on the aircraft radar bulkhead where the QS and the F-15B mate, such that when the FE model of the flight QS was validated, it could be readily mated to the aircraft FE model. This approach was established to enable the project to progress the model development and flutter predictions without the actual QS flight test hardware.

\section{Mathematical Background}

With the DFRC Mode Matching Code, three optimization phases are used to refine a model. The mass properties are modified to match measured mass properties, the mass matrix is orthogonalized, and then the natural frequencies and mode shapes are matched. Design variables for the optimization can include structural sizing information (thickness, cross sectional area, area moment of inertia, torsional constant, etc.), point properties (lumped mass, spring constant, etc.), and material properties (Young's modulus, etc.).

\section{Phase 1: Mass properties}

Matching mass properties by using lumped masses (CONM2) ${ }^{1}$ reduces the computational effort required to match stiffness properties and ensures that a physically meaningful and accurate solution is achieved. In this first optimization phase, ten objective functions are minimized to match analytical to measured mass properties. The mass properties that are updated include total mass, center of gravity location, and mass moments of inertia. As an objective function is minimized, a constraint function is implemented to prevent the updated variable from changing in the minimization of the following objective function. The constraint functions limit the amount of change allowed in the previously optimized variables. The objective functions and constraints applied in this phase are listed in order in Table 1. This optimization phase places the model within the vicinity of a physically viable solution (feasible region), facilitating the second and third optimization phases.

Table 1. Optimization Problem Statement for Phase 1 of Model Update

\begin{tabular}{|c|c|c|}
\hline Statement & Objective Function & Constraint Function \\
\hline 1 & $\mathrm{~J}_{1}=\left(\mathrm{W}-\mathrm{W}_{\mathrm{G}}\right)^{2} / \mathrm{W}_{\mathrm{G}}{ }^{2}$ & Unconstrained \\
\hline 2 & $\mathrm{~J}_{2}=\left(\mathrm{X}-\mathrm{X}_{\mathrm{G}}\right)^{2} / \mathrm{X}_{\mathrm{G}}{ }^{2}$ & $\left|\mathrm{~J}_{1}\right|<\varepsilon$ \\
\hline 3 & $\mathrm{~J}_{3}=\left(\mathrm{Y}-\mathrm{Y}_{\mathrm{G}}\right)^{2} / \mathrm{Y}_{\mathrm{G}}{ }^{2}$ & $\left|\mathrm{~J}_{\mathrm{i}}\right|<\varepsilon \quad \mathrm{i}=1,2$ \\
\hline 4 & $\mathrm{~J}_{4}=\left(\mathrm{Z}-\mathrm{Z}_{\mathrm{G}}\right)^{2} / \mathrm{Z}_{\mathrm{G}}{ }^{2}$ & $\left|\mathrm{~J}_{\mathrm{i}}\right|<\varepsilon \quad \mathrm{i}=1, \ldots 3$ \\
\hline 5 & $\mathrm{~J}_{5}=\left(\mathrm{I}_{\mathrm{XX}}-\mathrm{I}_{\mathrm{XXG}}\right)^{2} / \mathrm{I}_{\mathrm{XXG}}{ }^{2}$ & $\left|\mathrm{~J}_{\mathrm{i}}\right|<\varepsilon \quad \mathrm{i}=1, \ldots 4$ \\
\hline 6 & $\mathrm{~J}_{6}=\left(\mathrm{I}_{\mathrm{YY}}-\mathrm{I}_{\mathrm{YYG}}\right)^{2} \mathrm{I}_{\mathrm{YYG}}{ }^{2}$ & $\left|\mathrm{~J}_{\mathrm{i}}\right|<\varepsilon \quad \mathrm{i}=1, \ldots 5$ \\
\hline 7 & $\mathrm{~J}_{7}=\left(\mathrm{I}_{Z Z}-\mathrm{I}_{\mathrm{ZZG}}\right)^{2} / \mathrm{I}_{\mathrm{ZG}}{ }^{2}$ & $\left|\mathrm{~J}_{\mathrm{i}}\right|<\varepsilon \quad \mathrm{i}=1, \ldots 6$ \\
\hline 8 & $\mathrm{~J}_{8}=\left(\mathrm{I}_{\mathrm{XY}}-\mathrm{I}_{\mathrm{XYG}}\right)^{2} / \mathrm{I}_{\mathrm{XYG}}{ }^{2}$ & $\left|\mathrm{~J}_{\mathrm{i}}\right|<\varepsilon \quad \mathrm{i}=1, \ldots 7$ \\
\hline 9 & $\mathrm{~J}_{9}=\left(\mathrm{I}_{\mathrm{YZ}}-\mathrm{I}_{\mathrm{YZG}}\right)^{2} / \mathrm{I}_{\mathrm{YZG}}{ }^{2}$ & $\left|\mathrm{~J}_{\mathrm{i}}\right|<\varepsilon \quad \mathrm{i}=1, \ldots 8$ \\
\hline 10 & $\mathrm{~J}_{10}=\left(\mathrm{I}_{\mathrm{ZX}}-\mathrm{I}_{\mathrm{ZXG}}\right)^{2} / \mathrm{I}_{\mathrm{ZXG}}{ }^{2}$ & $\left|\mathrm{~J}_{\mathrm{i}}\right|<\varepsilon \quad \mathrm{i}=1, \ldots 9$ \\
\hline
\end{tabular}

Phase 2: Mass Matrix Orthogonalization

The orthonormalized mass matrix is shown in Eq. (1). 


$$
\underline{M}=\Phi_{G}{ }^{T} T^{T} M T \Phi_{G}
$$

The off diagonal terms of this orthonormalized matrix are reduced to improve mass orthogonality. In Eq. (1), the mass matrix, denoted as M, represents the analytical mass matrix, while $\Phi_{\mathrm{G}}$ represents the target Eigen matrix that is obtained from the GVT. The target Eigen matrix, $\Phi_{\mathrm{G}}$, remains unchanged during the optimization. The transformation matrix, $T$, is based on Guyan reduction ${ }^{2}$ or improved reduced system ${ }^{3}$. The optimization problem statement then becomes:

Minimize

$$
\left.J_{11} \equiv \sum_{i=1, j=1, i \neq j}^{n}\left(\underline{M}_{i j}\right)^{2} \quad \text { (Off-diagonal terms of } \underline{\underline{M}}\right)
$$

Such that $\left|\mathrm{J}_{\mathrm{i}}\right|<\varepsilon_{\mathrm{s}}$ where $\mathrm{i}=1,2 \ldots 10\left(\mathrm{~J}_{\mathrm{i}}\right.$ values are given in Table 1$)$, and $\mathrm{n}$ denotes the number of modes that will be correlated in Eq. (2). The positive-definiteness characteristic of the lumped mass elements also acts as a constraint during this optimization procedure.

\section{Phase 3: Frequencies and Mode Shapes}

In this phase, certain modes can be given a higher level of importance by applying a scaling factor $\beta$ in Eqs. (3) and (5). This scaling factor helps to more closely tune the frequencies of these analytical modes of interest after the mode shape vectors have been matched to within required accuracy.

Two optimization methods are used in this phase. In the first method, the objective function considered combines an index which identifies normalized errors between test $\left(\Omega_{\mathrm{i}}\right)$ and analytical $\left(\omega_{\mathrm{i}}\right)$ frequencies with a second index which defines the total error associated with the off-diagonal terms of the orthonormalized stiffness matrix. The optimization problem statement then becomes:

\section{Minimize}

$$
J_{12} \equiv \sum_{i=1}^{n}\left(\frac{\Omega_{i}-\omega_{i}}{\Omega_{i}}\right)^{2}+\beta \sum_{i=1, j=1, i \neq j}^{n}\left(\underline{K}_{i j}\right)^{2}
$$

Such that $\left|\mathrm{J}_{\mathrm{i}}\right|<\varepsilon$ for $\mathrm{i}=1,2 \ldots 11$. The matrix $\underline{\mathrm{K}}$ is a result of the matrix multiplication shown in Eq. (4), where the stiffness matrix, $\mathbf{K}$, is the analytical stiffness matrix.

$$
\underline{K}=\Phi_{\mathrm{G}}{ }^{\mathrm{T}} \mathrm{T}^{\mathrm{T}} \mathbf{K} \mathrm{T} \Phi_{\mathrm{G}}
$$

In the second optimization method, the objective function combines the same frequency error index used above, with a second index which defines the total error between test and analytical mode shapes at specified sensor locations. The optimization problem statement then becomes:

Minimize

$$
J_{12} \equiv \sum_{i=1}^{n}\left(\frac{\Omega_{i}-\omega_{i}}{\Omega_{i}}\right)^{2}+\beta \sum_{i=1}^{m}\left(\Phi_{i}-\Phi_{i G}\right)^{2}
$$

Such that $\left|\mathrm{J}_{\mathrm{i}}\right|<\varepsilon$ for $\mathrm{i}=1,2 \ldots 11$, and $\mathrm{m}$ denotes the number of degrees of freedom measured. The frequencies $\omega_{i}$ and mode shapes $\Phi_{i}$ are calculated based on the direct-iterative Eigen solution technique for large eigenvalue problems ${ }^{4}$ :

1) Compute $\mathbf{K}_{\mathrm{T}} \& \mathbf{M}_{\mathrm{T}}: \quad \mathbf{K}_{\mathrm{T}}=\mathrm{T}^{\mathrm{T}} \mathbf{K T} \& \mathbf{M}_{\mathrm{T}}=\mathrm{T}^{\mathrm{T}} \mathbf{M T}$

2) Compute $\mathrm{F}: \quad \mathrm{F}=\mathrm{M}_{\mathrm{T}} \Phi_{\mathrm{G}}$

3) Compute $R: \quad \quad F=K_{T} R$ 

4) Compute $K_{R} \& M_{R}: \quad K_{R}=R^{T} K_{T} R \& M_{R}=R^{T} M_{T} R$
5) Compute $\omega_{i}$ and $\Psi: \quad K_{R} \Psi=\omega^{2} M_{R} \Psi$
6) Compute $\Phi: \quad \Phi=\mathrm{R} \Psi$

For the direct-iterative Eigen-solution technique, the analytical stiffness and mass matrices, $\mathbf{K}$ and $\mathbf{M}$, are correlated, while the measured mode shapes, $\Phi_{\mathrm{G}}$, remain unchanged during the model updating process. Notice the transformation matrix $\mathbf{T}$ in Eqs. (1) and (4) is calculated using the analytical mass and stiffness matrices $\mathbf{M}$ and $\mathbf{K}$. Therefore, the constraint equation $\left|J_{11}\right|<\varepsilon$ is needed for the third optimization phase.

Current model updating approaches consist of updating only stiffness properties and assume that the modeled mass distribution is accurate. This can create erroneous stiffness properties in a model. DFRC's Mode Matching Code is capable of updating both mass and stiffness in a model by updating mass properties such as total mass, cg, and mass moments of inertia, improving the orthogonalization of the mass matrix, and matching frequencies and mode shapes to test data. Updating both mass and stiffness properties of a model will provide a higher degree of confidence in a model's resemblance of the actual hardware.

\section{Application}

An example of an application of the Mode Matching Code is the F-15B Research Testbed. NASA DFRC possesses a modified F-15B that is used as a test bed aircraft for supersonic flight experiments. Traditionally, the FE model of the flight test article is generated and updated by GVT data and then mated to the updated F-15B FE model. A GVT is then conducted with the test article mated to the aircraft. The mated FE model is correlated to this mated GVT data with the going-in assumption that, at this point, the only unknown variable is the interface stiffness between the test article and the aircraft.

Recently, Gulfstream Aerospace Corporation (GAC) desired to test a modified nose, the Quiet Spike ${ }^{\mathrm{TM}}$, on DFRC's F-15B'. Because of flight test article availability and a compressed project schedule, a non-traditional testing and model update method had to be implemented. A build-up approach was devised such that a Mock QS would be designed and fabricated to replace the flight QS in the tests and analyses. A detailed FE model of this Mock QS would be created. A reduced order model of this detailed FE model would be created in the form of a beam model to expedite the model update process faster. The beam model would then be matched to data provided by the first GVT using a strongback. This beam model would be mated to the F-15B model and the interface stiffness would be updated by a second GVT using the aircraft. The flight QS would be tested and its model would be updated separately at GAC. It would then be mated to the aircraft model and its modal analysis results would be compared to frequencies and mode shapes from a final GVT of the flight QS mated to the F-15B. Ultimately, the goal of this build-up approach was to completely eliminate the need for a model update of this final flight test configuration.

The flight QS measures $30 \mathrm{ft}$. in length when fully extended and $20 \mathrm{ft}$. when fully retracted. Figure 1 shows the GAC flight $Q S$.

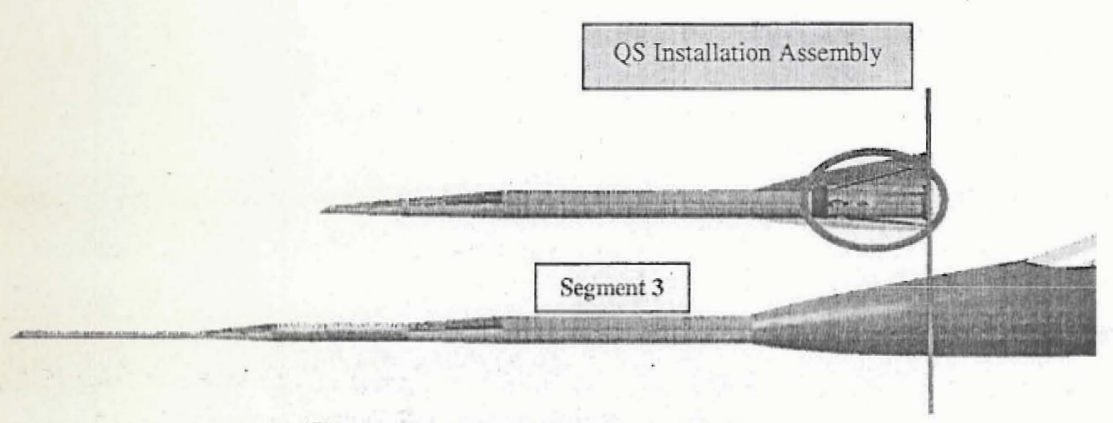

Figure 1. Flight QS Description

The Mock QS was designed with the purpose of replacing the flight QS in the build-up GVT approach. In order for the Mock QS to fulfill this purpose, it was necessary that its design meet the following requirements: 1) similar weight to flight QS weight, 2) similar CG to flight QS CG, and 3) similar $I_{x x}$ for the third segment of the flight QS. 
A final design requirement imposed on the Mock QS was that it was to interface with the strongback and F-15B radar bulkhead in the same manner and with the same hardware as the flight QS installation assembly. A study was done, which considered different materials, beam types, thicknesses, diameters, and lengths to determine the best design for the Mock QS. The Mock QS measures $19 \mathrm{ft}$ and is shown in Fig. 2.

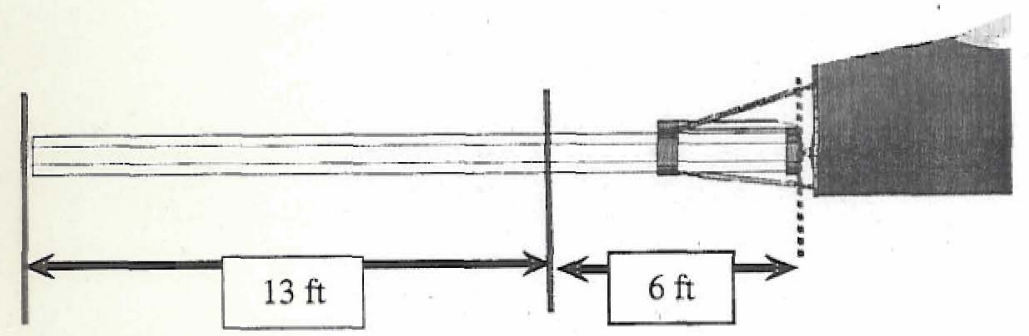

Figure 2. Mock QS Description

Several model updates coupled with a series of four GVTs were performed to acquire a correlated mated F15B/QS FE model that could be used in flutter analyses. Figure 3 shows the various models of the Mock QS that were developed to accomplish this task. The first FE model created was a detailed FE model of the Mock QS, which was created in Patran and MSC.Nastran using the Mock QS CAD models. It consisted of beam, plate, and solid elements and was fixed in a manner that represented how the flight QS mounted to the aircraft. A modal analysis was performed on the detailed FE model. The results from this modal analysis rendered the target frequencies and mode shapes for the beam model, which is shown on the far right in Fig. 3.

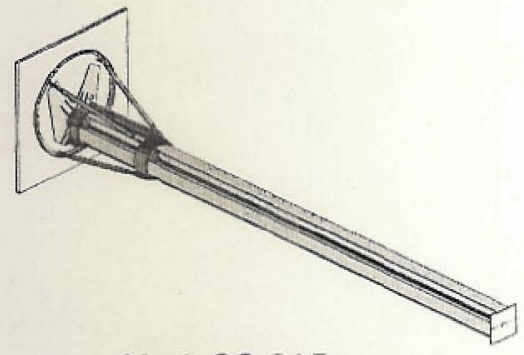

Mock QS CAD

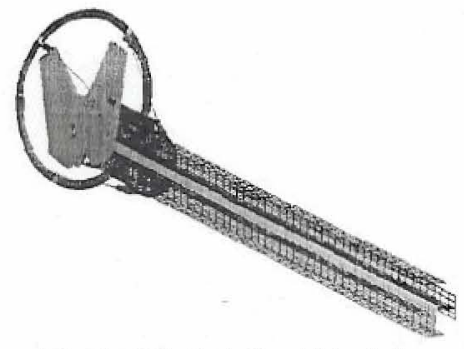

Mock QS Detailed Model

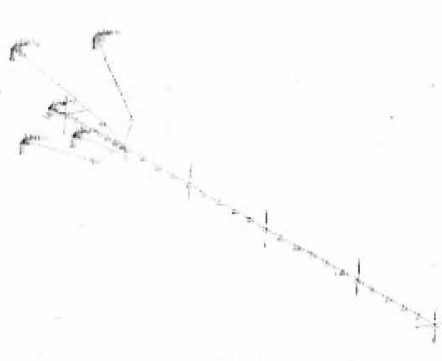

Mock QS Beam Model

Figure 3. Mock QS Model Development

The original beam model was developed in Patran and MSC.Nastran. The lumped mass and beam elements were created manually based on the numerical mass properties of the detailed model. Because the model update process requires much iteration, using a reduced order model decreases the computational effort required to match a model's structural properties to test data. This beam model decreased the computational effort in all analyses including the flutter analyses required at the conclusion of the model update process. Furthermore, matching the beam model to the detailed model first provided a good 'initial guess' for the Mock QS beam model and would, therefore, facilitate correlating the beam model to test data.

The next step in this task was to correlate the Mock QS beam model to the actual Mock QS structure. This was accomplished in the first GVT, which consisted of attaching the Mock QS boom to a strongback and exciting it in the lateral and vertical directions as shown in Fig. 4. The results from this GVT were used to update only the structural properties of the beam model. The interface stiffness was not updated until the second GVT. Attaching the Mock QS structure to the strongback provided a nearly rigid foundation for the boom, which ensured the boom modes were excited well. This also matched well with the boundary conditioned modeled. 


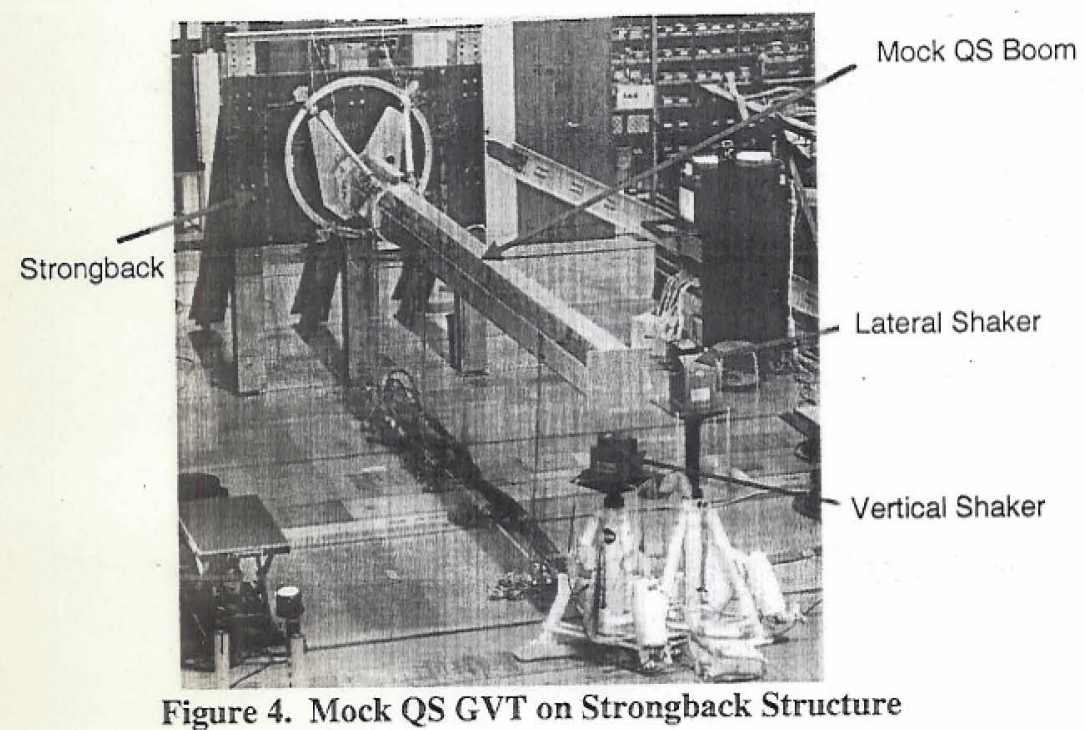

Figure 4. Mock QS GVT on Strongback Structure

When mating the updated Mock QS beam model to the updated aircraft beam model, it was assumed that any discrepancies between the numerical and the test mode shapes and frequencies could be attributed to the unknown connection stiffness between the test article and aircraft. The second GVT would empirically provide this connection stiffness. The aircraft was constrained laterally and vertically, such that the aircraft modes would be decoupled from the Mock QS modes. The Mock QS was again excited both laterally and vertically. This GVT configuration is illustrated in Fig. 5. Results from these GVT's are presented in Ref. 6.

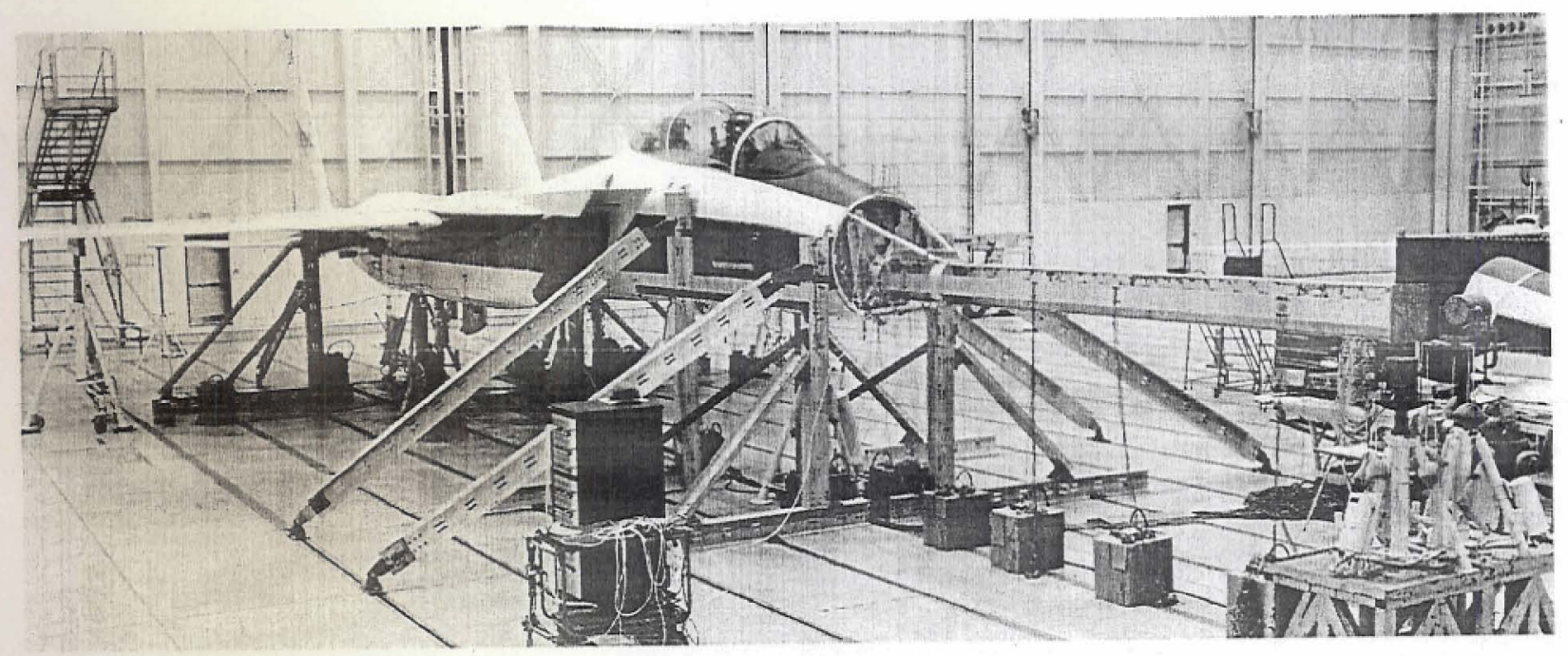

Figure 5. Mock QS Boom GVT on F-15B

The third GVT was the GVT of the flight QS on a strongback, which provided the modal parameters to update the flight QS model. The fourth and final GVT served as a final check for the mated F-15B/QS frequencies and mode shapes.

\section{Results and Discussion}

Because of the simplicity of the structure and the fidelity of the detailed FE model of the Mock QS, there was confidence in its representation of the actual Mock QS hardware. Therefore, in performing the first model update, 
the target modal values were provided by the numerical results of the detailed model to update the beam model. The first three modes of the detailed model or the target modes are shown in Fig. 6.

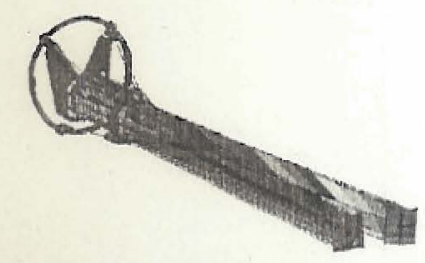

Mode 1-Lateral Bending $(5.675 \mathrm{~Hz})$

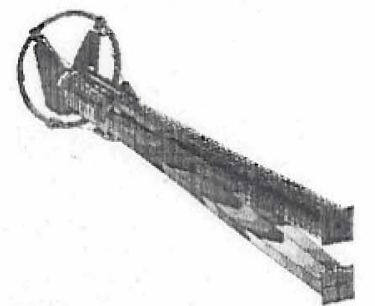

Mode 2-Vertical Bending $(6.602 \mathrm{~Hz})$

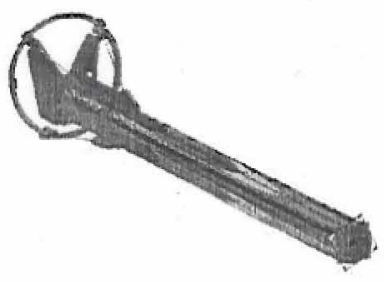

Mode 3-Torsion $(7.876 \mathrm{~Hz})$

Figure 6. Detailed Mock QS Mode Shapes and Frequencies

The first three modes of the detailed Mock QS were lateral bending at $5.68 \mathrm{~Hz}$, vertical bending at $6.6 \mathrm{~Hz}$, and torsion at $7.88 \mathrm{~Hz}$. Table 2 quantifies the similarity in the modal parameters between the beam and detailed Mock QS models before the beam model was updated.

Table 2. Comparison of Frequencies and MAC Values before Update

\begin{tabular}{|c|c|c|c|c|}
\hline Mode & Detailed FE Model $(\mathrm{Hz})$ & Beam Model $(\mathrm{Hz})$ & MAC $(\%)$ & Freq. Error $(\%)$ \\
\hline 1 & 5.675 & $7.285\left(3^{\text {rd }}\right)$ & 0.05 & 28.37 \\
\hline 2 & 6.602 & $7.515\left(1^{\text {st }}\right)$ & 0.02 & 13.83 \\
\hline 3 & 7.876 & $8.977\left(2^{\text {nd }}\right)$ & 0.03 & 13.98 \\
\hline
\end{tabular}

Table 2 indicates that, initially, the beam model did not correlate well with the detailed model. The beam model's modes were ordered differently - 1) torsion at $7.285 \mathrm{~Hz}, 2$ ) lateral bending at $7.515 \mathrm{~Hz}$, and 3) vertical bending at $8.977 \mathrm{~Hz}$. Consequently, this resulted in very low MAC values and high errors in frequency. The higher-than-expected frequencies imply that either the masses were underestimated or the stiffnesses were overestimated in the beam model.

As discussed in the previous section, the first phase of updating the beam model consisted of updating the mass properties. The optimization variables were assigned upper and lower limits of $+/-10$ per cent of the original values and included the mass values, cg's and inertias of the lumped mass elements. All mass properties were matched to within a half per cent error with the exception of the $y$-cg due to the detailed model possessing a $y$-cg at zero. A comparison for all mass properties is shown in Table 3.

Table 3. Comparison of Mass Properties after Update

\begin{tabular}{|c||c|c|c|c|c|c|c|c|c|}
\hline & Mass & $x-c g$ & $y-c g$ & z-cg & Ixx & Iyy & Izz & Iyz & Izx \\
\hline \hline Detailed & 400.01 & 114.67 & 0.00 & 108.08 & $2.60 \mathrm{E}+04$ & $2.16 \mathrm{E}+06$ & $2.15 \mathrm{E}+06$ & 7.40 & $2.41 \mathrm{E}+04$ \\
\hline Beam & 400.59 & 114.55 & 0.03 & 108.10 & $2.60 \mathrm{E}+04$ & $2.16 \mathrm{E}+06$ & $2.15 \mathrm{E}+06$ & 7.38 & $2.41 \mathrm{E}+04$ \\
\hline \hline Error & $-0.14 \%$ & $0.10 \%$ & $\mathrm{NaN}$ & $-0.02 \%$ & $-0.14 \%$ & $0.01 \%$ & $0.01 \%$ & $0.21 \%$ & $0.10 \%$ \\
\hline
\end{tabular}

The second phase of this model update consisted of producing an orthogonalized mass matrix. The variables were the same as in the previous phase. However, with the implementation of the constraint functions, the variables 
were limited to changing in such a way that the objective functions errors from the previous phase would not increase to a value greater than $\varepsilon$ (See Table 1). At the conclusion of this phase, the off-diagonal term in the mass matrix of the greatest value was 0.0135 . The resulting orthogonalized mass matrix was:

$\begin{array}{ccc}1.0000 & -0.2011 \mathrm{E}-03 & 0.1352 \mathrm{E}-01 \\ -0.2011 \mathrm{E}-03 & 1.0000 & 0.3827 \mathrm{E}-02 \\ 0.1352 \mathrm{E}-01 & 0.3827 \mathrm{E}-02 & 1.0000\end{array}$

The third phase of implementing DFRC's Mode Matching Code matches the beam model frequencies and mode shapes to the detailed model results. For this phase, the design variables were expanded to include element stiffness properties such as beam cross sectional area, area moment of inertias, and torsional constants. This set of design variables was also allowed to range $+/-10$ per cent to complete the model update procedure. Table 4 demonstrates how DFRC's in-house Mode Matching Code correlated the beam model to the detailed model.

Table 4. Comparisons of Frequencies after the First Update

\begin{tabular}{|c|c|c|c|c|}
\hline Mode & Detailed FE Model $(\mathrm{Hz})$ & Beam Model $(\mathrm{Hz})$ & MAC $(\%)$ & Freq. Error $(\%)$ \\
\hline 1 & 5.675 & 5.674 & 98.29 & -0.02 \\
\hline 2 & 6.602 & 6.607 & 98.14 & 0.08 \\
\hline 3 & 7.876 & 7.867 & 78.64 & -0.11 \\
\hline
\end{tabular}

The updated beam model showed good correlation to the detailed model. The first two mode shape vectors were matched to less than two per cent error, and the frequencies for all three modes were matched to within one per cent error. The third mode shape, which was the torsion mode, did not possess as good a MAC value as the first two. Because of the geometry of the QS and the additional torsional stiffness provided by the radome, the first torsion mode of the actual QS does not appear until about $100 \mathrm{~Hz}$. Therefore, it was deemed not critical to match the torsion mode with as much rigor as the lateral and bending modes, and a MAC value of 78 per cent sufficed for this mode.

Figure 7 shows the first three mode shapes of the Mock QS beam model after being updated.

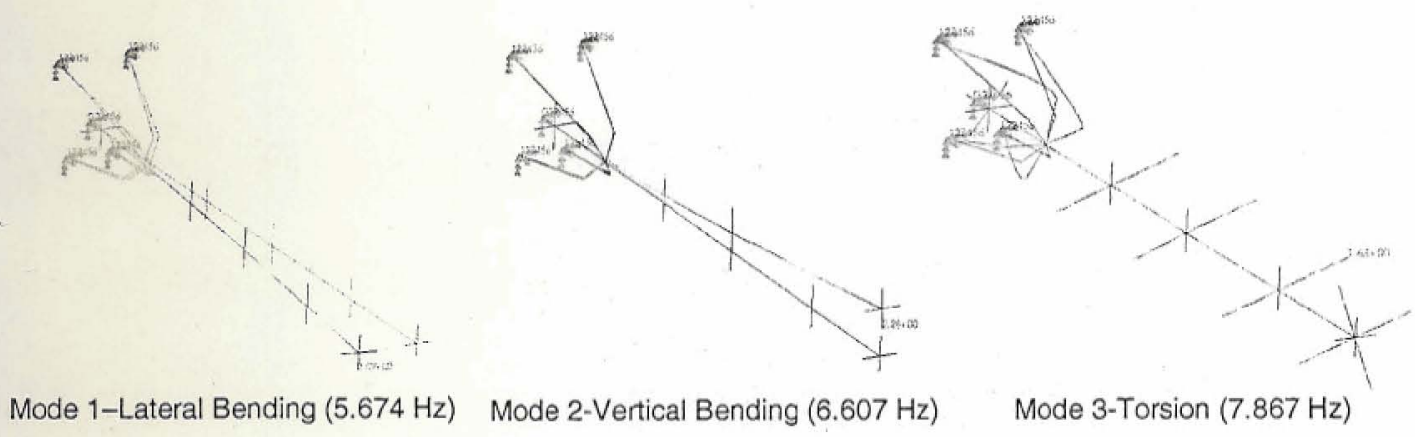

Figure 7. Mock QS Beam Model Mode Shapes and Frequencies

The beam model was next updated to match test data that resulted from the first GVT. This first Mock QS GVT required that the Mock QS be mounted to a strongback. The first three modes of the Mock QS were 1) lateral bending at $5.583 \mathrm{~Hz}, 2$ ) vertical bending at $7.752 \mathrm{~Hz}$, and 3) torsion at $8.105 \mathrm{~Hz}$. Updating the beam model to the detailed model first, made updating the beam model to test data faster and less computationally intensive. Table 5 compares the beam model to the strongback GVT results after only updating the mass properties. In this case, the only mass properties that were measured were total weight, and $\mathrm{x}-\mathrm{cg}$. The numerical values were kept for all other mass properties. 
Table 5. Comparison of Frequency and MAC Values at Update Start

\begin{tabular}{|c|c|c|c|c|}
\hline Mode & GVT $(\mathrm{Hz})$ & Beam Model $(\mathrm{Hz})$ & MAC $(\%)$ & Freq. Error (\%) \\
\hline 1 & 5.583 & 5.643 & 99.29 & $1.08 \%$ \\
\hline 2 & 7.752 & 7.529 & 99.49 & $-2.88 \%$ \\
\hline 3 & 8.105 & 8.142 & 67.66 & $0.45 \%$ \\
\hline
\end{tabular}

Because the beam model had already been through one update, its modal parameters already correlated well with the measured modal parameters. The design variables were treated similarly as before. They were assigned upper and lower limits of $+/-10$ per cent of the original values and included the mass values, cg's and inertias of the lumped mass elements. The total weight and $\mathrm{x}$-cg were updated to within a one per cent error.

The second phase of the model update, mass matrix orthogonalization, was also treated as previously done. The design variables were the same as in the previous phase. At the conclusion of this phase, the off-diagonal term in the orthogonalized mass matrix of the greatest value was 0.0737 . The resulting orthogonalized matrix was:

$\begin{array}{ccc}1.0000 & -0.1537 \mathrm{E}-01 & 0.7368 \mathrm{E}-01 \\ -0.1537 \mathrm{E}-01 & 1.0000 & -0.6174 \mathrm{E}-01 \\ 0.7368 \mathrm{E}-01 & -0.6174 \mathrm{E}-01 & 1.0000\end{array}$

Finally, the beam model was updated to have frequencies and mode shapes that matched the measured data. The design variables were again expanded to include element stiffness properties such as beam cross sectional areas, area moment of inertias, and torsional constants that were allowed to range $+/-10$ per cent. Table 6 shows how DFRC's in-house Mode Matching Code correlated the beam model to the actual Mock QS boom.

Table 6. Comparisons of Frequencies after Update to GVT Data

\begin{tabular}{|c|c|c|c|c|}
\hline Mode & GVT $(\mathrm{Hz})$ & FE Model $(\mathrm{Hz})$ & MAC (\%) & Error (\%) \\
\hline 1 & 5.583 & 5.594 & 99.25 & 0.20 \\
\hline 2 & 7.752 & 7.764 & 99.42 & 0.15 \\
\hline 3 & 8.105 & 8.093 & 88.51 & -0.15 \\
\hline
\end{tabular}

The resultant numerical modal parameters showed an excellent correlation to the measured modal parameters for the Mock QS test article. The first two mode shape vectors were matched to less than one per cent error, and the frequencies for all three modes were matched to within a half per cent error. Regarding the third mode shape, we see the same results as before. This mode, which was the torsion mode, did not possess as good a MAC value as the first two. Again, this mode was regarded as not being of great importance.

The final model update required that only the spring element stiffnesses that connected the Mock QS hardware to the aircraft be updated. Initially, they were assumed to be nearly rigid and therefore, were assigned high values. The second GVT would provide their true values. The second GVT consisted of mating the Mock QS boom to the F-15B. The aircraft was constrained vertically by supporting it with aircraft jacks and laterally by use of an erector set. This configuration decoupled the aircraft modes from the boom modes. The measured modes and frequencies of this configuration were lateral at $6.06 \mathrm{~Hz}$ and vertical at $7.75 \mathrm{~Hz}$. The torsion mode was not extracted for this configuration. Table 7 computes the frequency errors between the numerical and test modes.

Table 7. Comparison of Test and Numerical Modes of the Mock QS/F-15B

\begin{tabular}{|c|c|c|c|}
\hline Mode & GVT $(\mathrm{Hz})$ & FE Model $(\mathrm{Hz})$ & Freq. Error (\%) \\
\hline 1 & 6.064 & 5.583 & $8.62 \%$ \\
\hline 2 & 7.756 & 7.752 & $0.05 \%$ \\
\hline
\end{tabular}


The first aircraft test mode was at a higher frequency than the first numerical mode by almost half a Hertz. This was not expected, because the mated model already assumed a nearly rigid connection between the Mock QS and the aircraft. The higher frequency of the first aircraft test mode indicates that the aircraft was stiffer laterally than the strongback, which can be explained by the high inertia of aircraft compared to the strongback and the erector set providing a true lateral constraint. This also indicates that the assumption of a nearly rigid connection was accurate. This is further supported by the fact that the second numerical mode frequency already matched the second test mode frequency to within a tenth of a per cent.

After the flight QS model was updated by test data, it was mated to the F-15B model using this nearly rigid connection stiffness. The project then proceeded to perform flutter analyses using this mated model. This final mated model matched well to GVT data, and therefore, no model update was necessary, and the flutter analysis did not need to be redone. The project could quickly proceed to the flight testing portion of this project.

\section{Conclusions}

NASA DFRC has developed an in-house Mode Matching Code that updates both the mass and stiffness properties of a model. This method renders a more accurate model over the method of only updating stiffness properties. The three phases of optimization that it employs are 1) updating mass properties, 2) orthogonalization of mass matrix, and 3) updating frequency and mode shape. The code has been applied in the GAC QS project flown on DFRC's F-15B supersonic flight test bed. The build-up approach used to update the QS/F-15B mated model was completed using this code. During this project, it was used to perform two model updates. It successfully updated each model used for each mode of interest to within one per cent error. Having used this code to update these models enabled this project to proceed with flutter analyses, such that when the flight test article, the project could quickly move into flight testing.

\section{References}

${ }^{1}$ Reymond, M. and Miller, M., MSC/NASTRAN Quick Reference Guide version 69, The MacNeal-Schwendler Corporation, 1996.

${ }^{2}$ Guyan, R. J., "Reduction of Stiffness and Mass Matrices." AIAA Journal, Vol. 3, No. 2, 1965.

${ }^{3} \mathrm{O}$ 'Callahan, J. C., "A Procedure for an Improved Reduced System (IRS) Model." 7th International Modal Analysis Conference, pp. 17-21, Las Vegas, Jan. 1989.

${ }^{4}$ Dong, S. B., Wolf, J. A., and Peterson, F. E., "On a Direct-Iterative Eigensolution Technique," International Journal of Numerical Methods in Engineering, Vol.4, pp. 155-162, 1972.

${ }^{5}$ Simmons, F., Freund, D., Spivey, N., and Schuster, L., "Quiet Spike ${ }^{\mathrm{TM}}$ : The Design and Validation of an Extendable Nose Boom Prototype", AIAA-2007-1774, April 2007 (to be published)

${ }^{6}$ Spivey, N., Herrera, C., Truax, R., Pak, C., and Freund, D, "Quiet Spike ${ }^{\mathrm{TM}}$ Build-Up Ground Vibration Testing Approach", AIAA-2007-1775, April 2007 (to be published) 